\title{
The effect of the square root of time on continuing perceptual tasks'
}

\author{
M. M. TAYLOR \\ DEFENCE RESEARCH MEDICAL LABORATORIES, TORONTO, CANADA
}

When an observer is confronted with a stimulus pattern that in some aspect does not change over time, perception of that aspect of the pattern does change. This paper documents several different types of change, all of which progress linearly with the square root of the observing time. Examples are drawn from studies of figural after-effects, motion aftereffects, vigilance, motion neutralization, visibility of the stabilized retinal image, effects of contours on visibility and fluctuations in the perceptual organization of ambiguous figures.

Rarely is the human perceiving system confronted with a stimulus pattern that does not change over time in all its major features. Even if the external environment is unchanging, the eyes rove, the head and body move, so that the observer continually induces more or less complex transformations in the incoming patterns of stimuli. The perceiving mechanism processes this continuously altering mass of information, to produce a relatively permanent and stable model of the external world.

In experimental situations, the human observer may be deprived of the opportunity to observe stimulus patterns transformed by his actions. One extreme form of experiment in which the opportunity is restricted is the sensory deprivation experiment, in which the subject is denied as far as possible coherent visual, auditory or kinaesthetic input. Results typically include distortion of perception and illusory movement of objects when normal conditions are restored. The effects of less drastic reductions in the ability of the observer to transform his environment, and in the rate of alternation of the environment, have been studied. Experiments with partially or completely stabilized retinal images come under this heading, as do studies on vigilance, and studies of the behavior of ambiguous or reversing figures with continued observation. Perceptual aberrations of one sort or another seem to be a common result of all such experiments, and in many of them the change in perception seems to progress linearly with the square root of the time on task. The purpose of the present paper is to present a number of examples of such experiments

\section{Figural After-Effect}

The phenomena subsumed under the name of "figural after-effect" are many and varied. The effect that will be considered here is a simple displacement effect. Observation of a stimulus with a defined position on some perceptual dimension causes perception of a subsequently observed stimulus with a different position on that dimension to be displaced away from the percept of the first stimulus. Other effects that are sometimes included as figural after-effects are changes in the first (inspection) figure, or perhaps alterations due to the act of judging the second (test) figure. Such effects are not considered here.

A typical experiment of the class giving figural aftereffect as considered here might use a concentric circle figure. A circle displaced from the fixation point is inspected for some period, after which the display is replaced by one with two circles, a test circle concentric with the inspection circle and a comparison circle on the opposite side of the fixation point. If the test circle is smaller than the inspection circle, it is typically judged as equal to a comparison circle which would have been judgt $A$ to be smaller had the inspection circle not been presented. This alteration in the apparent size of the test circle is known as the figural after-effect displacement of perceived size. Similar effects have been observed with many different geometric figures and with non-geometric properties of visual stimuli, as well as in various sensory modalities.

It has been proposed, with reasonable predictive success (Taylor, 1962, 1963a) that the displacement effect is due to changes in the discriminability of parts of the continuum following observations of the inspection figure. Three major assumptions were proposed (Taylor, 1962). They read, in part, as follows:

(1) "... The perceived distance between two points is a monotonic increasing function of the discriminability of the difference between them, other factors remaining constant."

(2)"... The introduction of a point into an interval of perceptual space will alter the discriminability of that interval: if the point is clearly distinguished from all pre-existing points, discriminability will be increased, but if it enters appreciably into confusion with any other point, discriminability may be decreased."

(3) ".. The observer will use information from all available sources about an event, giving the information from each source a weight inversely related to the variability of the source."

These assumptions were given quantitative expression in subsidiary assumptions of linearity of relations and were used to predict the magnitude of the after-effect. In particular, the magnitude of the after-effect was related directly to the precision of location of the 

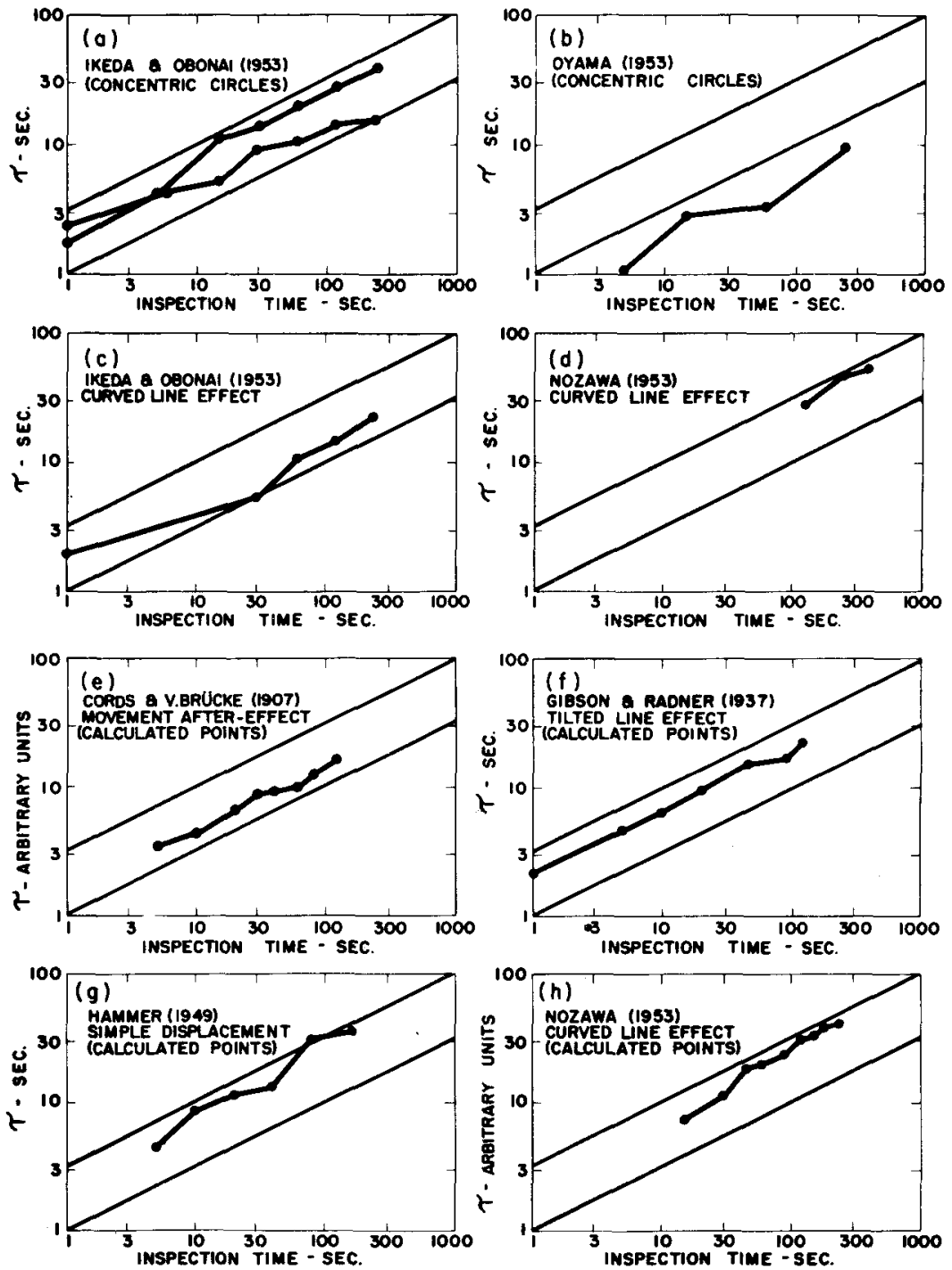

Fig. 1. The relation of inspection period to the decay time constant for various experiments on figural aftereffects. The top four graphs represent direct measurement, the lower four, calculated values. Note that the units for the decay time constant are arbitrary in (e) and (h). Data from Taylor (1962) reprinted from Canad. J. Psychol. inspection figure at the time of test. It was determined, from a consideration of a number of studies and the reanalysis of data from others that the effectordinarily decayed exponentially, with a time constant proportional to the square root of the length of the inspection period. The initial effect appears to be independent of the inspection period for the figures used in these experiments, provided that the inspection period is longer than about $100 \mathrm{msec}$. (Suto \& Ikeda, 1957, and others cited by Taylor, 1962). The experimental results for the decay time constant $(r)$ are shown in Fig. 1 . The straight lines on each graph have a slope of $1 / 2$, so that a line of experimental points parallel to them would represent a square root relation between the time constant and the inspection period. In general, the directly measured points do run roughly parallel to the straight lines. The calculated points were taken from experiments in which a single determination was made for each inspection period, some time after the end of the inspection period, so that the effect had time to decay before measurement. Assumption of an asymptotic value for infinite inspection time allowed computation of a decay time constant for each point separately. Details are given in the original paper (Taylor, 1962). The computed points are in agreement with those measured directly, supporting the suggestion of a square root relation between inspection period and the decay time constant.

The same theoretical considerations that apply to the static effects apply also to the continuum of motion. In other words, the motion after-effects should be subject to the same laws as the static effects. Direct tracking of the course of decay of a rotary motion aftereffect suggests that the initial effect is dependent on the inspection period, and that the time constant does not increase as the square root of the inspection period over the whole range of times examined. This result may be understood within the same theoretical frame- 


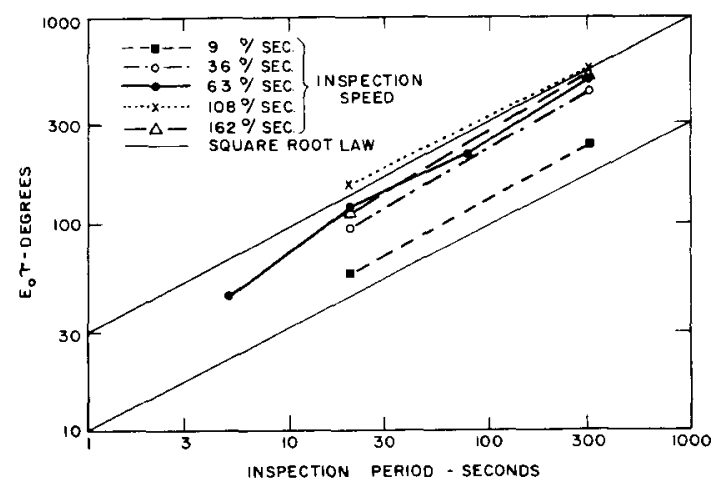

Fig. 2. The relation between the integrated after-effect of seen rotary movement and the inspection time for different speeds of the inspection disc. Data from Taylor $(1963 b)$ reprinted with permission from Percept. mot. Skills.

work if it is considered that the precision of observation of speed increases with observation of time over periods of up to a minute (Taylor, 1963b).

Although neither the initial after-effect nor the decay time constant obey the laws applicable to the static after-effects directly, the integrated after-effect (given by their product) does. Both for the static effect and for the motion after-effect, the product of time constant and initial after-effect varies as the square root of the inspection period. Figure 2 shows the integrated rotary motion after-effect as a function of time for five different inspection speeds. Again the straight lines have a slope representing a square root relation.

The fact that the integrated after-effect is proportional to the square root of the inspection period suggests that successive samples of the stimulus input are equally weighted in the integration, since the amount of after-effect is assumed to vary inversely with the standard deviation of the observation of the inspection figure. This standard deviation should be inversely proportional to the square root of the number of samples contributing to its determination, i.e., of the time spent observing, if the samples are equally precise.

The remaining examples of the square root dependence of perceptual effects on inspection time show that this presumption is not strictly true. The deviation appears not sufficient, however, to be detectable in the experiments represented in Figs. 1 and 2. While the figural after-effect may be considered an integrative effect, in that it depends on the total information processed during the inspection period, the remaining effects may mostly be considered as "instantaneous" effects, since they are concerned with the continued gathering of information, whose value depends on the prior inspection.

\section{Vigilance}

The most direct test of the constancy of perceptual efficiency with time on task is a determination of the detectability of some small chang in the stimulus parameters. When a measurement of detection is repeated over a reasonably long period of continued observation, the experiment is generally termed a "vigilance" experiment.

Using a stimulus consisting of a brief pause in the motion of the sweep second hand of a clock, Mackworth and Taylor (1963) have measured the detectability, $d^{\prime}{ }_{\text {, }}$ of the signal over watch periods of up to an hour. Different methods of responding and groups of subjects from different populations were used in the study, which was based on earlier work by Mackworth (1963). The detectability of the signal declined continuously over the observation period. Figure 3 shows the change of detectability of the signal in some of the experiments from the Mackworth and Taylor study. The detectability measure was d', a measure which has been shown to be relatively independent of the observer's willingness to guess about the existence of the signal (for example, Swets et al (1961)). In each case, the detectability decreased exponentially (linearly, on the logarithmic plot of Fig. 3), with the square root of inspection time, and with approximately the same time constant, slope, for all conditions. The rate of change of detectability was low, about $0.03 \mathrm{log}$ units $/ \sqrt{\mathrm{min} .}$, so that the integrated detectability would not have been very different from that predicted without the decline, over $4 \mathrm{~min}$., the longest period used in the experiments of Fig. 1.

Data reported by Wiener, Poock, and Steele (1964) on a vigilance task, were reanalyzed by Taylor (1965) in terms of detectability measures, with the result that the detectability of the signal was not impaired by the passage of time. Rather, the criterion applied by the subject changed, again linearly with the square root of time. Figure 4 shows the change in criterion over time for Wiener, Poock, and Steele's three experimental groups.

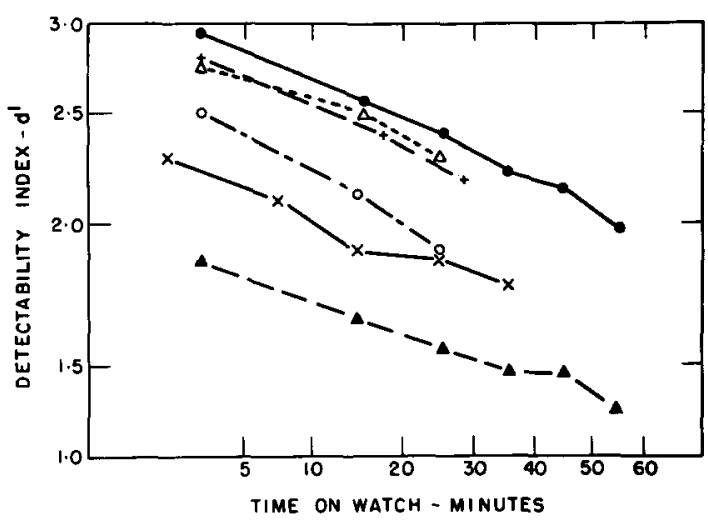

Fig. 3. Relation between detectability of a signal and time on watch. Time scale is square root. All signals are short pauses in the movement of a sweep second hand of a clock. The separare curves represent data from independent experiments of the same type. Data from Mackworth and Taylow (1963). 


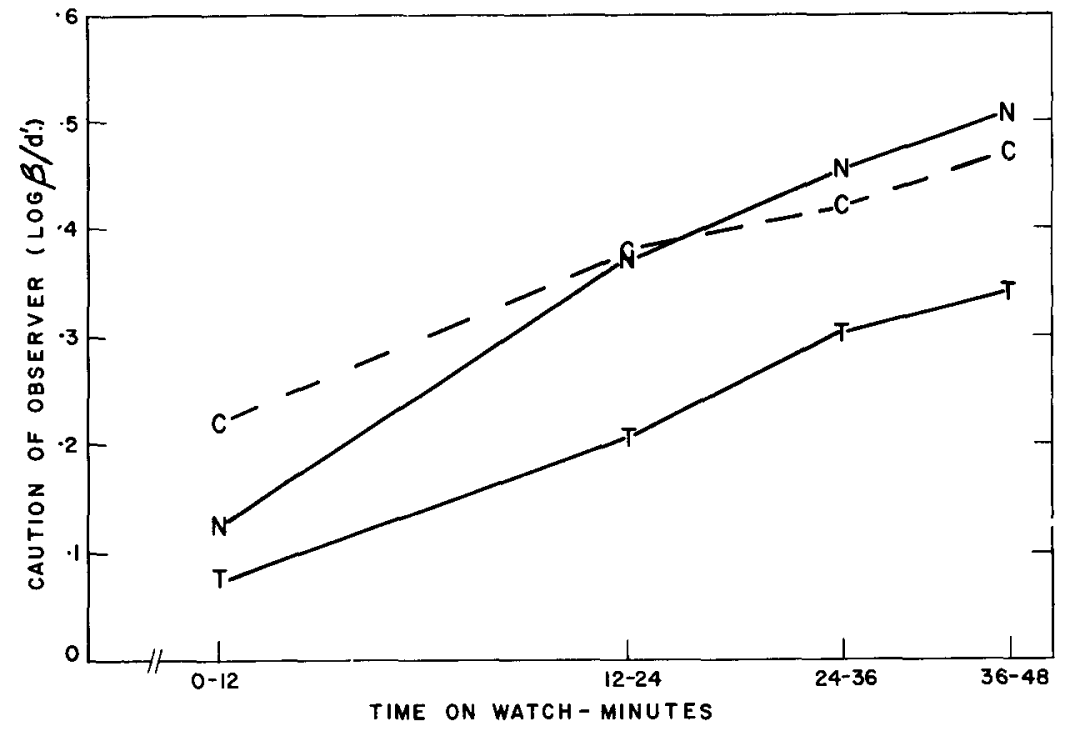

Fig. 4. Increase in caution $\left(\log \beta / d^{\prime}\right)$ for three groups of subjects. $T, C, N$ refer to different experimental conditions irrelevant to the present discussion. Data from Wiener, Poock, and Steele (1964) as reanalyzed by Taylor (1965), reprinted with permission from Percept. mot. Skills.
The difference between this study and those discussed above seems to be in that Wiener, Poock, and Steele required their subjects to detect a variant presentation of otherwise identical repetitive events, whereas Mackworth (1963) and Mackworth and Taylor (1963) asked for detection of signals superimposed on a continuous background. Mackworth (1965) has shown that for discrete background (flashes of light), detectability does not change over time when the background rate is slow (less than one flash per second) but does when the background rate is fast. Wiener, Poock, and Steele used a slow background rate.

\section{Movement Neutralization}

According to Assumption 1 of the figural after-effect theory, if the discriminability of the difference between a constantly observed speed and zero decreases, then so should the apparent speed. Such a decrease in apparent speed with continued observation has been reported by Gibson (1937). This neutralization does not show complete interocular transfer, if indeed it shows any, so that it may readily be observed by examining a steadily moving stimulus pattern with one eye for some period, and then changing to the other eye. The pattern will then seem to move much faster.

The neutralization of rotary movement has been studied by a compensatory tracking technique (Taylor, 1963c). The subjects were shown a disc, which then started to rotate at some predetermined speed. Using a control, they were required to keep the disc rotating at its initial speed, compensating for nonexistent changes introduced by the experimenter. The actual speed of the disc, as adjusted by the subject, increased exponentially with the square root of time, during the period of watch. Figure 5 shows the logarithmic increase of speed averaged over all conditions and subjects,

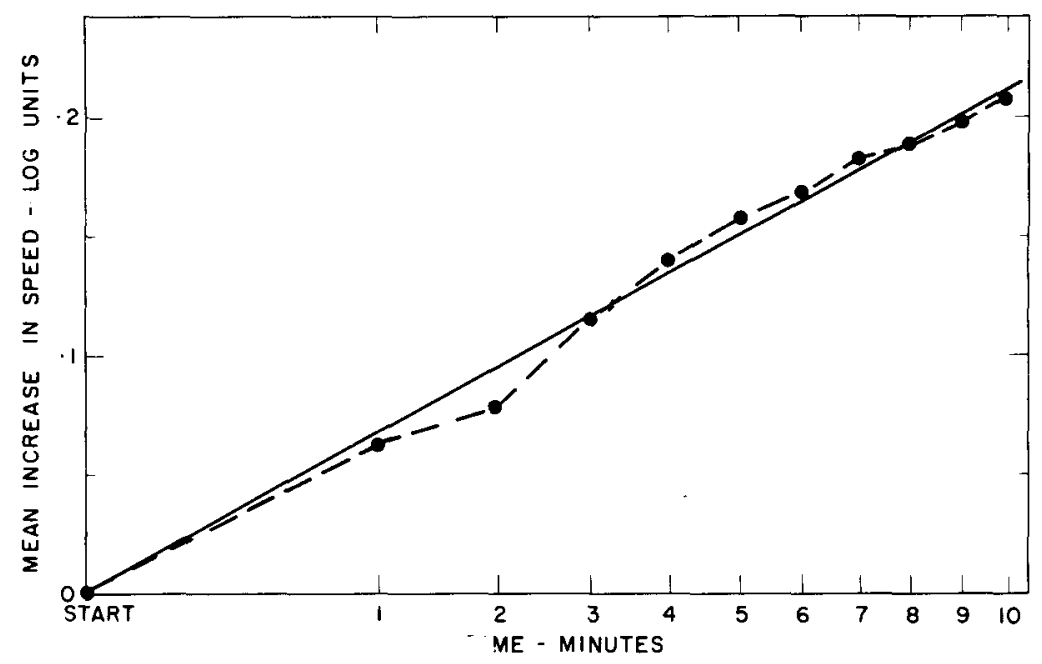

Fig. 5. Logarithmic increase in rotary speed seen as equal to initial speed, plotted against the square root of time on task. Data from Taylor $(1963 c)$ reprinted with permission from Percept. mot. Skills. 


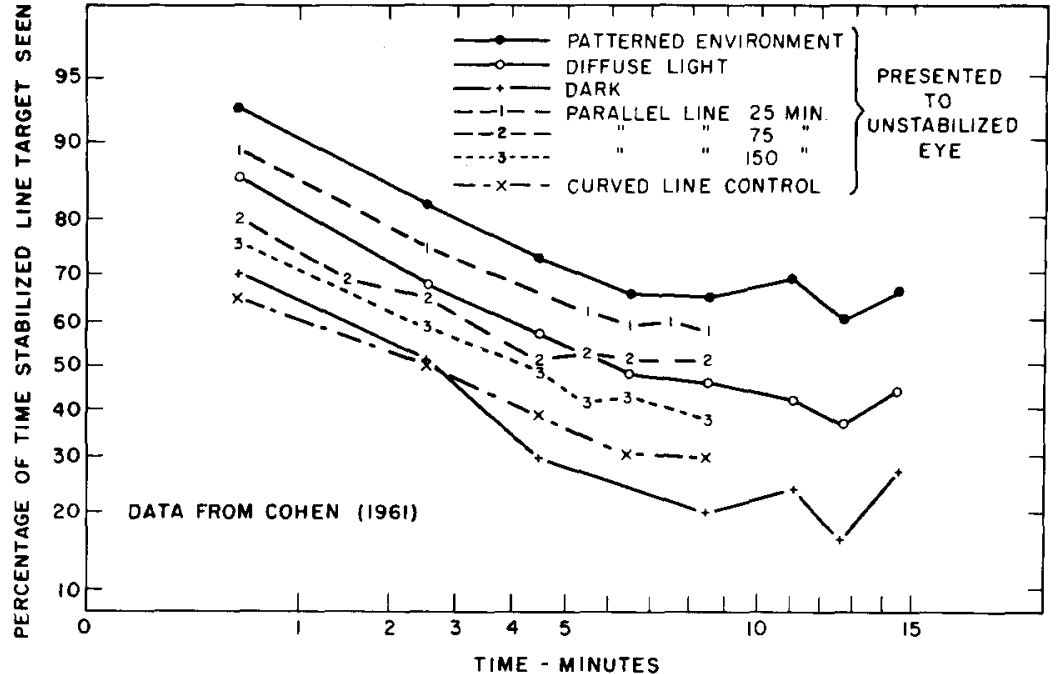

Fig. 6. Percentage of time a retinally stabilized line target is seen with different presentations to the other eye, plotted on probability paper against the square root of time. Data from Cohen (1961). plotted against the square root of the time since the start of the inspection. The average rate was about $0.07 \log$ units $/ \sqrt{\mathrm{min}}$., with a range, over the conditions studied, from 0.04 to 0.12 . Large individual differences in rate were noted, but the square root law held for each subject.

\section{Visibility of a Partially Stabilized Retinal Image}

It has often been observed that when an image is stabilized on the retina by use of a system including a contact lens, the percept quickly disappears, only to reappear and disappear irregularly. Barlow (1963) has pointed out that these erratic fluctuations of the percept are probably caused by movements of the contact lens over the eye. He claims that well stabilized images never reappear in their original contrasty form, but sometimes appear in a milky manner and they do not reappear at all after about a minute of observation. Accordingly, the reappearances reported for images stabilized for periods longer than a minute must be due to lens slippage and destabilization of the image, as must all the contrasty reappearances reported. The course of the fluctuations may then be described in terms of two components. In the initial phase the image would fluctuate even with perfect stabilization, presumably because of some remanent effect of the first sight and to these fluctuations the regenerations due to slippage are added. In the second phase, only the regenerations due to slippage occur.

Cohen (1961) has investigated the proportion of time a "stabilized" straight line target is seen, as a function of the length of inspection, with different unstabilized targets presented to the other eye. His results seem to confirm the two-phase description of the course of the fluctuations. In Fig. 6, Cohen's data are shown replotted on a probability scale against the square root of inspection time. These data, from one subject, seem to be well fitted by an almost straight line of negative slope which approaches a cc:stant value asymptotically about $7 \mathrm{~min}$. after the viewing period begins. The difference between the curves may be interpreted as due to differences in intrinsic visibility of the target under the different conditions.

The curves of Fig. 6 do not support the contention that the square root of time is the controlling variable so strongly as do those presented earlier, because of the asymptotic character of the data after the seventh minute. A plot of the Cohen data even against log time provides reasonable approximations to straight lines, but the approximations are not as good as those given by the square root plot.

\section{Effect of a Contour on Visibility of Nearty Light Spot}

Following the work of Yokose and his collaborators (e.g. Yokose, 1954), Nozawa ( 58) has investigated the change of threshold for a light spot exposed near a previously fixated dark contour on a light field. When the contour is a straight line, the threshold for the spot is elevated, and the amount of elevation continues to increase with continuing observation of the line. When the contour is a circle, what happens to the threshold depends on the location of the spot relative to the circle. If it is outside, it becomes easier to see. For spots equally distant from the contour, the change in threshold seems to be well described by a component due to the mere presence of the contour-i.e.: the straight line effect-plus a component due to the curvature of the contour, positive for regions inside the curve, and negative for regions outside. As Fig. 7 shows, both portions of the effect progress with the square root of the inspection time. Obviously, the improvement in visibility cannot continue indefinitely, so that the straight line relationship must eventually break down in that case at least. 


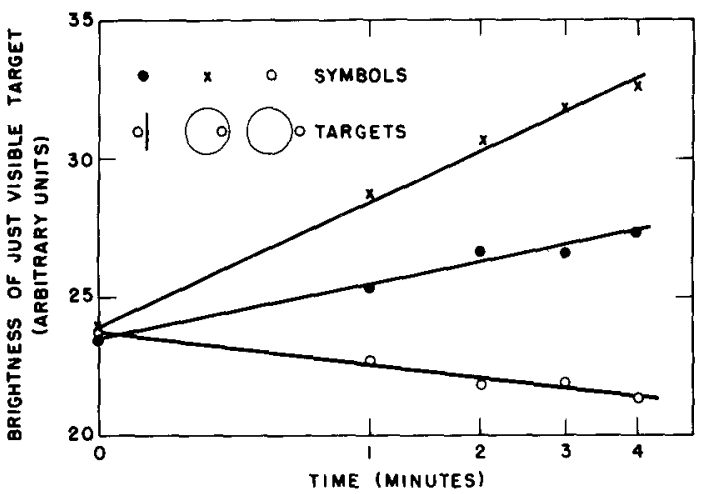

Fig. 7. Change in brightness of just detectable spot of light following inspection of dark contour on light field, for different forms of contour. Time scale is square root. Data from Nozawa (1958).

\section{Fluctuations of Perceptual Organization}

The foregoing four examples all involved a change in sensitivity to a stimulus. But continued observation of a stimulus pattern results not only in reduction of sensitivity, but also in perceptual alterations. The ubiquitous nature of this effect has been noted by Sakurabayashi $(1952,1953)$ using both auditory and visual material. Numerical data have been obtained by Warren (1961a, 1961b) for the number of different forms reported as a function of time for the verbal transformation effect, and by Taylor and Henning (1963a, $1963 \mathrm{~b})$ for the same effect as well as for other static and dynamic visual figures and for an auditory beep sequence. Figure 8 shows a representative sample of these results, in which the cumulative numbers of novel forms (forms not including the first form seen) are plotted against the square root of the time on task. In general, the relation is linear, but novel forms do not occur until a short while after the start of the observation. Different rates apply for the different tasks but, in general, after about 0.25 to $0.5 \mathrm{~min}$. the number of novel forms increases with the square root of observation time.

\section{Comment}

It is not at all clear why the perceptual efficiency of the human organism should decrease linearly with the square root of time. That it should decrease makes adaptive sense. When the stimulus input is highly correlated from moment to moment, the informative value of later samples is low, and there is little gain to the perceiving mechanism in maintaining highest precision. If the information processing capacity of any or all sub-units of the perceiving mechanism can be deployed in various ways, then it is reasonable that the portion conceded to the redundant input should be reduced, allowing greater caparity for other purposes. The consistency of the decline over so varied a range of perceptual tasks suggests that it is fairly general property of natural information processing devices, at least in such an adaptable machine as the human.

\section{References}

Barlow, H. B. Slippage of contact lenses and other artefacts in relation to fading and regeneration of supposedly stable retinal images. Quart. J. exp. Psychol., 1963, 15, 36-51.

Cohen, H. B. The effect of contralateral visual stimulation on visibility with stabilized retinal images. Canad. J. Psychol. $1961,15,212-219$.

Mackworth, J. F. The effect of intermittent signal probability upon vigilance. Canad. J. Psychol., 1963, 17, 82-89.

Mackworth, J. F. Deterioration of signal detectability during a vigilance task as a function of background event rate. Psychon. Sci., 1965, 3, 421-422.

Mackworth, J. F., \& Taylor, M. M. The d'measure of signal detectability in vigilance-like situations, Canad. J. Psychol., 1963, $17,302-325$.

Nozawa, S. An experimental study on figural after-effect by the measurement of field strength, II. Jap. psychol. Res., 1958, 1, $5,22-27$.

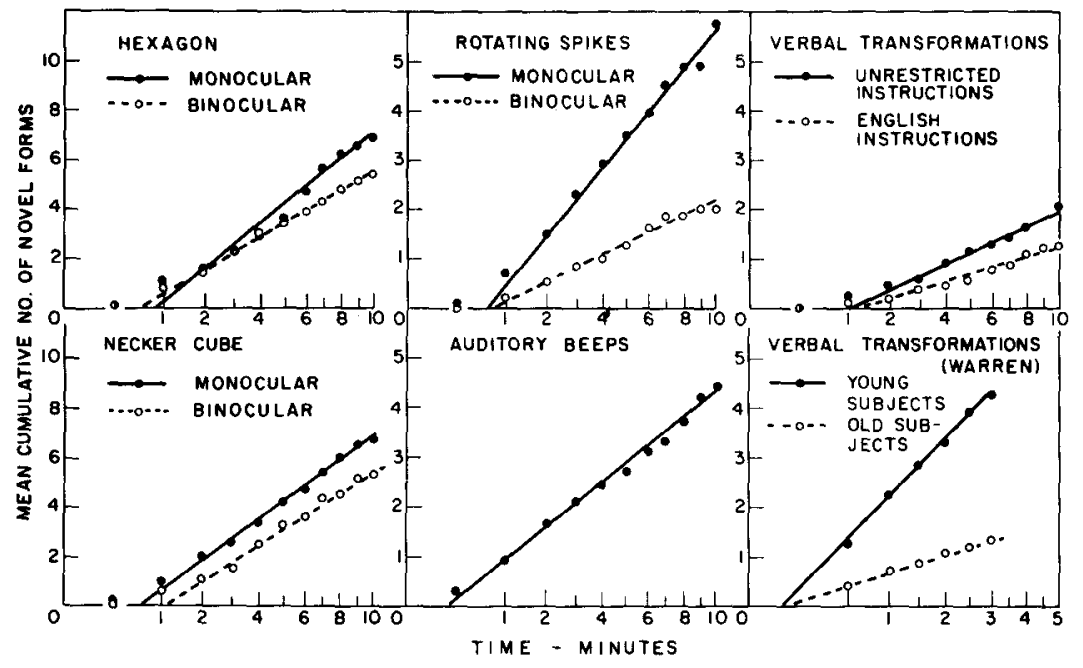

Fig. 8. Mean cumulative number of novel forms reported as a function of time watching or listening to an unvarying stimulus pattem. Time scale is square root. Data from Warren (1961a; 1961b) and from Taylor and Henning 1963a; 1963b). 
Sakurabayashi, H. Study in creation III: the formation of auditory gestalts with repeated sound stimuli and their developments. Jap. J. Psychol., 1952, 23, 12-25.

Sakurabayashi, H. Studies in creation IV: the meaning of prolonged inspection from the standpoint of creation. Jap. J. Psychol., $1953,23,207-216$.

Suto, Y., \& Ikeda, H. An examination of the relationship between the inspection time and the figural after-effects. Jap. J. Psychol., 1957, 27, 377-380.

Swets, J. A., Tanner, W. P., \& Birdsall, T. G. Decision processes in perception. Psychol. Rev., 1961, 68, 301-340.

Taylor, M. M. Figural after-effects: a psychophysical theory of the displacement effect. Canad. J. Psychol., 1962, 16, 247-277.

Taylor, M. M. Numerical prediction of a simple figural after-effect as a function of the contrast of the inspection figure. Psychol. Rev., 1963a, 70, 357-360.

Taylor, M. M. Tracking the decay of the after-effect of seen rotary movement. Percept. mot. Skills, 1963b, 16, 119-129.

Taylor, M. M. Tracking the neutralization of seen rotary movement. Percept. mot. Skills, 1963a, 16, 513-519.

Taylor, M. M., \& Henring, G. B. Verbal transformations and an effect of instructional bias on perception. Canad. J. Psychol., 1963a, 17, 210-223.
Taylor, M. M., \& Henning, G. B. Iransformations of perception with prolonged observation. Canad. J. Psychol., 1963b, 17, 349-360.

Taylor, M. M. Detectability measures in vigilance: comments on a paper by Wiener, Poock, and Steele. Percept. mot. Skills, 1965 , $20,1217-1221$.

Warren, $\mathbf{R}$. M. Illusory changes in repeated words: differences between young adults and the aged. Amer. J. Psychol., 1961a, 74, 505-516.

Warren, R. M. Illusory changes of distinct speech upon repetition the verbal transformation effect. Brit. J. Psychol., 1961b, 52, 249-258.

Wiener, E. L., Poock, G. K., \& Steele, M. Effect of time sharing on monitoring performance: simple mental arithmetic as a loading task. Percept. mot. Skills, 1964, 19, 435-440.

\section{Note}

1. Review Paper 512. This paper, with the exception of the note on Wiener, Poock and Steele's experiment, was presented at the AGARD Symposium on Natural and Artificial Logic Processors, Athens, July, 1963.

(Received in the Editorial Office March 2, 1966.) 